Cahiers $d u$ MONDE RUSSE

\section{Cahiers du monde russe}

Russie - Empire russe - Union soviétique et États indépendants

$43 / 4 \mid 2002$

Intellectuels et intelligentsia

\title{
Ronald Grigor Suny, Terry Martin, eds, A state of nations
}

Juliette Cadiot

\section{OpenEdition}

\section{Journals}

Édition électronique

URL : https://journals.openedition.org/monderusse/4046

DOI : 10.4000/monderusse.4046

ISSN : $1777-5388$

Éditeur

Éditions de l'EHESS

\section{Édition imprimée}

Date de publication : 30 décembre 2002

Pagination : 768-770

ISBN : 2-7132-1796-2

ISSN : $1252-6576$

Référence électronique

Juliette Cadiot, "Ronald Grigor Suny, Terry Martin, eds, A state of nations », Cahiers du monde russe [En ligne], 43/4 | 2002, mis en ligne le 17 juin 2009, consulté le 03 septembre 2022. URL : http:// journals.openedition.org/monderusse/4046; DOI : https://doi.org/10.4000/monderusse.4046

Ce document a été généré automatiquement le 3 septembre 2022

Tous droits réservés 


\title{
Ronald Grigor Suny, Terry Martin, eds, A state of nations
}

\author{
Juliette Cadiot
}

\section{RÉFÉRENCE}

Ronald Grigor SUNY, Terry MARTIN, eds, A state of nations. Empire and nationmaking in the age of Lenin and Stalin. Oxford-New-York, Oxford University Press, $2001,307 \mathrm{p}$.

1 Cette série d'articles, rédigés pour la plupart par de jeunes historiens, donne un large aperçu du renouveau de l'historiographie américaine contemporaine sur la question des nationalités en Union soviétique. L'objet de l'ouvrage est ambitieux : il s'agit d'écrire une nouvelle histoire de l'URSS, définie par son caractère multiethnique. C'est dans une volonté de rupture, voire de changement de paradigme, que les nouvelles études sur la dimension multinationale de l'Union soviétique se placent, en cherchant à caractériser l'État soviétique comme n'étant ni un empire, ni un État-nation, mais une entité politique originale qui entreprit de promouvoir systématiquement les minorités nationales. La qualification de l'Union soviétique comme empire de discrimination positive (The affirmative action empire), inventée par Terry Martin dans son ouvrage du même nom, sert ici de fil conducteur aux différentes études, monographiques ou thématiques, comme au choix des bornes chronologiques (de la révolution aux années 1950).

2 Mais, plus que l'illustration d'une thèse, ce livre offre l'avantage de présenter une série de réflexions et d'études très diverses, utilisant des méthodologies variées, allant de la science politique à l'histoire des sciences sociales, ou encore à l'histoire culturelle, inspirée par les études post-coloniales. Il prouve ainsi combien le fait de placer la dimension multiethnique de l'URSS au centre de l'attention des historiens est fructueuse et permet d'en renouveler non seulement l'histoire politique, mais aussi l'histoire sociale et culturelle. 
3 La richesse de ces matériaux soulève de très nombreuses questions qui traversent l'ensemble de l'histoire du $\mathrm{xx}^{\mathrm{e}}$ siècle, par exemple en ce qui concerne la dimension coloniale de l'URSS, les différentes formes de discrimination ethnique et de lutte envisageables contre ces dernières ou encore la création des États-nations.

4 L'article de Ronald Grigor Suny se situe dans la période présoviétique. Il y définit le contenu donné au concept d'empire à travers différentes époques historiques. Celui de Terry Martin illustre et justifie sa formule qualifiant l'URSS d'empire de discrimination positive, qui se manifesta par la promotion, voire la création, de territoires, langues, cultures et élites non russes. Il retrace les débats qui agitèrent les élites bolcheviques juste après la révolution quant à la place à accorder aux revendications nationalistes, et souligne en particulier l'engagement de Stalin et Lenin en faveur de la reconnaissance des droits des minorités nationales.

5 La seconde partie de l'ouvrage réunit des articles qui interrogent la rupture révolutionnaire. Surtout, ils introduisent une autre dimension du politique, à travers l'étude de nouveaux acteurs sociaux, agents étatiques ou militants politiques, porteurs de projets spécifiques. Joshua Sanborn distingue la notion d'ethnos national de celle de national pour souligner que le projet de création d'une société civile s'est articulé bien plus autour de l'utilisation de métaphores familiales que proprement ethniques. Étudiant principalement les discours et pratiques de l'armée, il montre combien cette dernière a mobilisé et contrôlé ses troupes grâce à l'utilisation d'une rhétorique s'appuyant sur les liens familiaux. J. Sanborn ne pousse cependant pas plus loin sa réflexion sur la création d'une communauté politique par l'instauration de relations directes entre l'État et les individus, à travers les politiques de la famille. Peter Holquist offre, lui aussi, une problématique nouvelle et très stimulante, liée ici explicitement à une réflexion sur l'État et ses pratiques d'intervention sur la société. Il s'interroge plus particulièrement sur le rôle des statistiques et des penseurs militaires dans les politiques de terreur (déportation et extermination) entreprises par l'empire comme par l'URSS contre tel ou tel peuple, en particulier les Tchétchènes. Holquist introduit une réflexion comparative, et dans le temps long, sur les technologies issues des sciences humaines visant à décrire les populations comme autant d'entités élémentaires, infiniment manipulables.

6 Les études suivantes sont des monographies qui s'intéressent aux projets et évolutions politiques des élites ou acteurs locaux, directement issus des minorités nationales. Adeeb Khalib décrit les positions politiques des Jadidistes et leurs transformations au fur et à mesure des événements révolutionnaires. Il offre une vision dynamique et complexe du projet «nationaliste» des élites intellectuelles d'Asie centrale ainsi que de leur accord avec la conception ontologique de la nation et les critiques anti-impérialistes des bolcheviks. L'article de Daniel E. Schafer montre combien l'institutionnalisation des nationalités en URSS fut aussi et surtout le résultat d'une série de micro-événements, de négociations pragmatiques. Il s'intéresse en particulier au problème de la territorialisation, en décrivant par le détail la naissance de la république du Bashkortostan et nous montre comment les nationalistes bachkirs passèrent par opportunisme politique du côté des Blancs à celui des bolcheviks, dans le but de se faire reconnaître un territoire. Schafer en conclut que la politique nationale soviétique, bien qu'obéissant aux schémas tracés par Terry Martin, s'est construite dans la guerre par des initiatives locales, auxquelles le Centre répondait avec beaucoup d'improvisation. Douglas Northrop s'intéresse, lui encore, au nationalisme local, mais cherche cette fois à en interroger l'ambigu contenu culturel. Il décrit de quelle façon le voile des femmes en 
Ouzbékistan, du fait des pratiques discursives russes, est devenu un symbole national ouzbek et comment la politique de libération des femmes engagée par le régime ne pouvait être comprise que comme impérialiste. Illustrant le problème des pratiques discriminatoires et des stéréotypes à l'encontre des Kazakhs chez les ouvriers et cadres russes du chantier ferroviaire du Turksib, Matt Payne cherche dans les pratiques à déterminer si l'Union soviétique fut bel et bien un empire de discrimination positive. Il envisage ainsi l'importance de l'héritage des pratiques coloniales russes et l'ambiguïté de discours qui débouchèrent finalement sur la russification des ouvriers kazakhs.

7 La dernière partie du livre concerne la fin de la période stalinienne et s'interroge précisément sur la possibilité d'appliquer le modèle de Terry Martin sur la longue durée, alors même qu'un discours russo-centrique se met en place à l'approche de la guerre. Peter Blitstein revient sur la décision prise en 1938 d'introduire le russe comme langue subsidiaire obligatoire dans les écoles non russes, mesure souvent interprétée comme rompant avec la politique de reconnaissance et de promotion de la diversité nationale de l'URSS. Il en conclut que ce ne fut qu'après la mort de Stalin qu'une politique systématique de russification fut entreprise. Dans son étude sur le travail de propagande des historiens pendant la Seconde Guerre mondiale, David Brandenberger analyse l'ambiguïté du nationalisme russe à la fin de la guerre. La rupture russo-centrique serait en définitive moins la remise en cause de l'empire de discrimination positive qu'une entreprise de construction de l'État, centrée sur la puissance russe, mais ne remettant pas en cause l'existence et les droits des autres nationalités.

8 La dimension comparative n'est que partiellement explorée dans l'ouvrage, les deux éditeurs ayant surtout cherché à qualifier et à dégager la spécifité de l'URSS, sans toujours la relier à un ensemble de problématiques historiques plus générales. Le titre paradoxal de l'ouvrage et la discussion autour des notions d'empire et d'État-nation, ainsi que l'absence de réflexion d'ensemble sur l'État, sont moins convaincants que le contenu des articles. Enfin une introduction permettant de faire un bilan plus nuancé de l'avancée des recherches, des différents courants d'analyse, mais surtout des très nombreuses études empiriques à mener, comme des questions encore en suspens et des sources à explorer, aurait sans doute été plus stimulante. 